\title{
Removal of Intensity Bias in Magnitude Spin-Echo MRI Images by Nonlinear Diffusion Filtering
}

\author{
Alexei A. Samsonov", Chris R. Johnson \\ Scientific Computing and Imaging Institute, University of Utah, $50 \mathrm{~S}$ Central Campus Drive, \\ Room 3490, Salt Lake City, UT, 84112, USA
}

\begin{abstract}
MRI data analysis is routinely done on the magnitude part of complex images. While both real and imaginary image channels contain Gaussian noise, magnitude MRI data are characterized by Rice distribution. However, conventional filtering methods often assume image noise to be zero mean and Gaussian distributed. Estimation of an underlying image using magnitude data produces biased result. The bias may lead to significant image errors, especially in areas of low signal-to-noise ratio (SNR). The incorporation of the Rice PDF into a noise filtering procedure can significantly complicate the method both algorithmically and computationally. In this paper, we demonstrate that inherent image phase smoothness of spin-echo MRI images could be utilized for separate filtering of real and imaginary complex image channels to achieve unbiased image denoising. The concept is demonstrated with a novel nonlinear diffusion filtering scheme developed for complex image filtering. In our proposed method, the separate diffusion processes are coupled through combined diffusion coefficients determined from the image magnitude. The new method has been validated with simulated and real MRI data. The new method has provided efficient denoising and bias removal in conventional and black-blood angiography MRI images obtained using fast spin echo acquisition protocols.
\end{abstract}

Keywords: MRI, anisotropic diffusion filtering, vector-valued diffusion, fast spin echo, Rice distribution, intensity bias

\section{INTRODUCTION}

MRI images are complex-valued and contain zero-mean uncorrelated Gaussian noise in both real and imaginary image parts. Such noise could be efficiently eliminated using filtering procedures based on spatial averaging. In practice, image analysis is often performed on magnitude data, as the image magnitude is not susceptible to variations characteristic for image phase ${ }^{1}$. Magnitude data are characterized by Rice probability density function (PDF) ${ }^{2}$. Though spatial averaging produces biased results for Rice distributed data, the estimation methods based on Gaussian PDF are still preferred, as the inclusion of the Rice PDF into a filtering procedure usually complicates it both algorithmically and computationally ${ }^{3}$. While for high SNR areas the bias is not significant, it becomes more pronounced for low SNR regions. This systematic error might present a serious problem in studies where contrast-to-noise ratio (CNR) in low SNR areas is essential (i.e. black-blood angiography ${ }^{4}$ ), or in quantitative evaluations of T1/T2 maps ${ }^{5}$.

One way to avoid the biased results is to filter real and imaginary parts separately. This would eliminate the Gaussian noise and produce unbiased estimation of the complex image channels and, consequently, the image magnitude. However, while MRI image magnitude generally satisfies ergodicity assumption ${ }^{6}$, nonsmooth image phase variations generally introduce rough modulations into both real and imaginary image channels breaking the assumption. Aside from other acquisition approaches, MRI acquisition techniques based on spin-echo phenomenon ${ }^{1}$ create images with smooth and slowly varying image phase. This is due to the refocusing properties of the spin-echo effect. The phase smoothness is used in partial Fourier methods ${ }^{7}$ that use low-frequency phase estimates in reconstructing a reduced set of asymmetrically sampled $k$-space data. The phase introduces smooth modulations in the intensities of the complex image channels that could be handled by many nonlinear filtering approaches ${ }^{8,9}$ to realize the idea of separate channel filtering.

\footnotetext{
*samsonov@sci.utah.edu; phone 1801 585-0052; fax 1801 585-6513; http://www.sci.utah.edu; Scientific Computing and Imaging Institute, University of Utah, 50 S Central Campus Drive, Room 3490, Salt Lake City, Utah 84112
} 
In this paper, we demonstrate this idea using a novel nonlinear filtering scheme based on an anisotropic diffusion approach $^{10}$. The anisotropic diffusion filter is an iterative denoising technique used for MRI image enhancement ${ }^{11}$. The technique provides efficient removal of noise in homogeneous tissue areas while retaining image structures. Our method uses the inherent phase smoothness property of spin-echo images to fulfill the separate filtering of complex image parts. The separate diffusion processes are coupled through combined diffusion coefficients found using the image magnitude. In the next sections, we first give an overview of concepts related to the subject of the paper, namely, the PDF of MRI magnitude data, anisotropic diffusion filtering in Perona-Malik formulation ${ }^{10}$, and properties of spin-echo images. We then describe the new method and provide implementation details. We present results of testing the method on simulated, phantom and patient brain data. Finally, we discuss the advantages and limitations of the new technique.

\section{THEORY}

\subsection{PDF of MRI image magnitude}

MRI data are collected in the spatial-frequency domain, or $k$-space. A common way to reconstruct data sampled on the Cartesian grid is to transform the data into the spatial domain using a fast Fourier transform (FFT). Resulting image is complex-valued, and both real and imaginary parts are described by a Gaussian PDF. The image magnitude is computed as

$$
M=\sqrt{\operatorname{Re}(I)^{2}+\operatorname{Im}(I)^{2}},
$$

where $\operatorname{Re}(I)$ and $\operatorname{Im}(I)$ are real and imaginary parts of image $I$ correspondingly. The nonlinear magnitude operation leads to the Rice PDF of the image magnitude ${ }^{2,12}$ :

$$
p_{M}(M \mid \mu, \sigma)=\frac{M}{\sigma^{2}} e^{-\frac{M^{2}+\mu^{2}}{2 \sigma^{2}}} I_{0}\left(\frac{\mu M}{\sigma^{2}}\right) \mathcal{E}(M),
$$

where $\mu$ represents the true image amplitude, $\sigma$ is the standard deviation (STD) of the data, $I_{0}($.$) is the 0^{\text {th }}$ order modified Bessel function of the first kind, and $\varepsilon($.) is a unit step function indicating that the PDF is valid only for non-negative values of $M$. If more than two Gaussian variables are used to calculate image magnitude, then a generalized Rice PDF ${ }^{2}$ should be used instead. The Rice PDF for several values of SNR defined as $\mu / \sigma$ is plotted in Figure 1. For high SNR $(\mathrm{SNR}>3)$, the Rice PDF approximates the Gaussian PDF, and for low SNR (SNR<3) it approximates a Rayleigh distribution:

$$
p_{M}(M \mid \sigma)=\frac{M}{\sigma^{2}} e^{-\frac{M^{2}}{2 \sigma^{2}}} \varepsilon(M)
$$

The Rayleigh distribution characterizes samples in air background areas where $\mathrm{SNR}=0$. It is common practice to estimate the image noise STD using relations ${ }^{13}$

$$
\sigma_{b g}=0.655 \cdot \sigma
$$

and

$$
\mu_{b g}=1.253 \cdot \sigma
$$

where $\sigma_{b g}$ and $\mu_{b g}$ is the STD and the mean of samples in air background areas.

The bias relative to the amplitude $\mu$ vs. SNR is depicted in Figure 2. As could be appreciated from the plot, the bias is low SNR areas is comparable with image amplitude. There could be significant degradation of local CNR defined as the difference in SNR between two tissue types. CNR is one of the most important characteristics of diagnostic quality of MRI images. Accuracy of disease diagnosis is strongly dependent on the difference in signal strength between normal and pathological tissue types. 


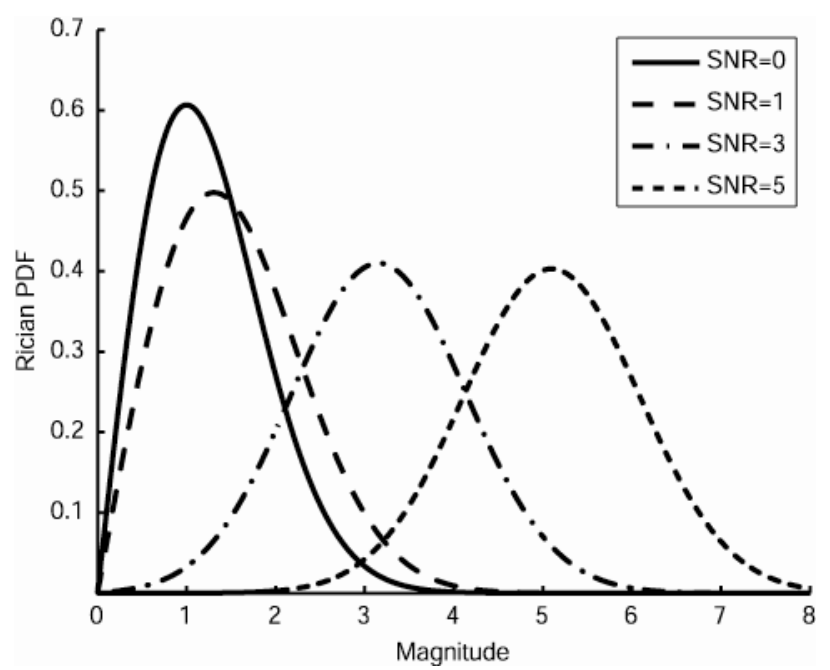

Figure 1: Rice PDF for several values of SNR.

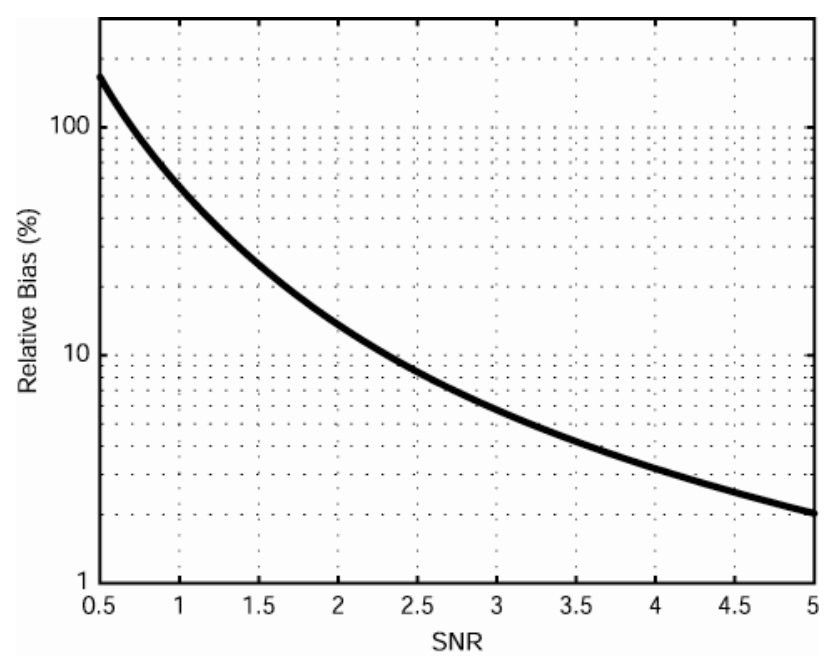

Figure 2: Relative bias of spatial averaging signal estimation vs. SNR. The bias is independent of the number of averaged pixel values and completely determined by image SNR.

\subsection{Anisotropic diffusion filtering}

A nice property of anisotropic diffusion filtering is that it preserves important image structures while removing noise. This is achieved by smoothing the image along object boundaries and preventing diffusion across the boundaries. Anisotropic diffusion filtering is equivalent to solving the following partial differential equation with respect to image function $I(\bar{r}, t)$ :

$$
\frac{\partial I(\bar{r}, t)}{\partial t}=\nabla(g(\|\nabla I\|, k) \cdot \nabla I(\bar{r}, t)),
$$

where $g(\|\nabla I\|, k)$ is a monotonically decreasing diffusivity function that depends on the value of the local image gradient $\nabla I$ and conductance parameter $k$, and $t$ is an artificial time parameter. In this work, we used an exponential diffusivity function ${ }^{10}$

$$
g(\|\nabla I\|, k)=\exp \left(-(\|\nabla I\| / k)^{2}\right)
$$

The parameter $k$ sets a threshold between image gradients to be smoothed and the ones to be preserved. The choice of the conductance parameter is crucial for the filter performance. In practice, a good choice for MRI data is ${ }^{11}$

$$
1.5 \sigma \leq k \leq 2 \sigma
$$

The parameter $\sigma$ could be estimated using analysis of air background samples (Eqs. [4, 5]).

In practice, many images such as color images and multiple contrast MRI data are vector-valued functions. A simple way to extend the anisotropic diffusion to vector-valued images is to simply solve Eq. [6] separately for each image channel. However, this approach does not work very well when diffusion coefficients are different for each image component ${ }^{14}$. Flexibility of the formulation based on the anisotropic diffusion (Eq. [6]) offers various opportunities to control the nonlinear filtering through a choice of diffusion coefficients. Most anisotropic diffusion schemes use common diffusion coefficients for all image components. This allows preservation of correlating and contrasting effects among multiple image channels ${ }^{11,15}$. This approach has been taken in application of the anisotropic diffusion filtering to multi-echo MRI data ${ }^{11}$, where diffusion coefficients were calculated using a combined image gradient measure. 


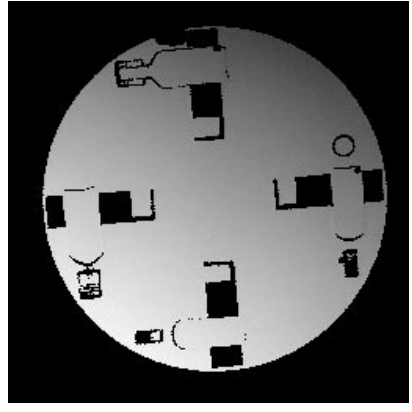

$\mathbf{a}$

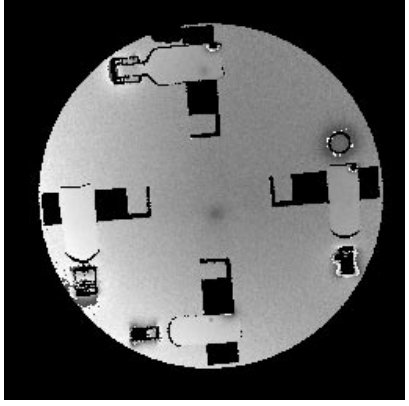

b

Figure 3: Image phase for (a) FSE and (b) gradient echo (GRE) scans of the same phantom object. The phases are shown in the limits of object mask obtained by thresholding image magnitude. Note smooth behavior of FSE image phase and rapid phase variations on edges of the object for GRE image phase.

\subsection{Phase properties of spin-echo MRI images}

MRI images should be real valued, if the ideal experiment conditions are met. However, in practice, the images are complex. Phase variations in the images occur due to the various factors such as a noncentered sampling window, patient-to-patient variations in coil loading, inhomogeneity in RF pulse spin-flipping angle, eddy currents, main field inhomogeneities, susceptibility, and chemical shift effects ${ }^{1}$. The first three factors create global, slowly varying phase changes, while the last three are responsible for fast phase transitions. Spin-echo effect used to form MRI signal eliminates the spin dephasing that is due to main field, susceptibility and chemical shift effects and prevents the corresponding phase variations in MRI image. As a result, image phase for spin-echo acquisitions is described by a smooth slowly varying function (Fig. 3a). For gradient-echo-based (GRE) sequences, the behavior of the phase is more complicated (Fig. 3b). In this work, we make use of the phase smoothness to separate real and complex image channel filtering. Due to this property, real and imaginary parts of complex spin-echo MRI images are well described by a piecewise slowly varying model (Fig. 4).

The phase smoothness property is used in partial Fourier reconstruction ${ }^{7}$ of spin-echo data that makes use of Hermitian symmetry of the Fourier transform. In this approach, the $k$-space is sampled asymmetrically to reduce the

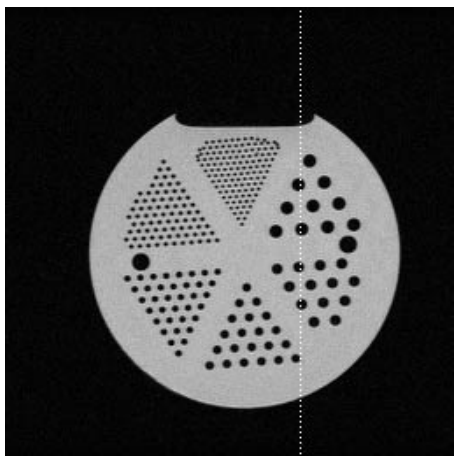

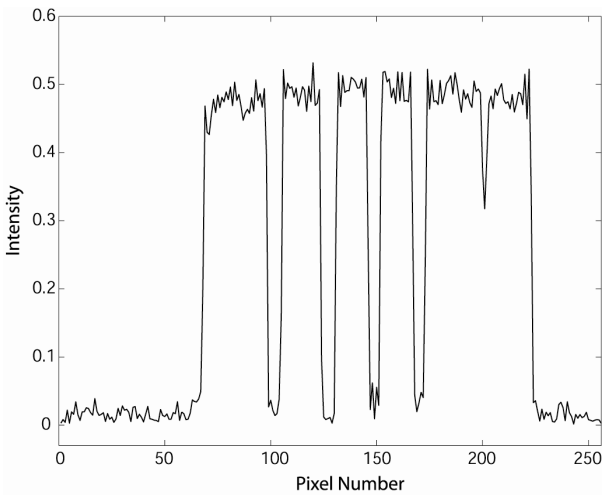

b

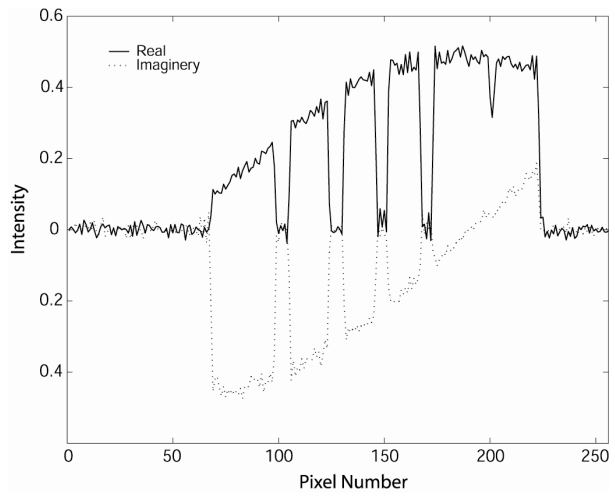

c

Figure 4: Magnitude (a) of a phantom image, and plots of image magnitude (b) and real and imaginary image parts (c) using the profile indicated in (a). 
amount of data needed for image reconstruction and, consequently, achieve the scan time reduction. The symmetrically sampled k-space center is used to obtain low-frequency estimate of image phase. The missing data are then filled in using phase correction algorithms, for example, POCS-based reconstruction ${ }^{16}$. The algorithms are known to produce artifacts in areas where true image phase significantly deviates from its low-resolution phase estimate. These variations are due to magnetic susceptibility changes on air-tissue boundaries and blood flow effects ${ }^{1}$.

\section{METHODOLOGY}

The method we present in the paper is a particular case of a vector-valued diffusion ${ }^{15}$. The image channels are smoothed separately. At the same time, the same diffusion coefficients are utilized to ensure the consistency of the separate diffusion processes. To calculate the coefficients, diffusion stopping gradients are found from the image magnitude. The main rationale for using this scheme is that the image magnitude provides a more reliable representation of tissue structures than the real and imaginary image channels in separate. The corresponding diffusion equation to be solved has the form of Eq. [6]. However, the image function $I(\bar{r}, t)$ is now complex-valued, and the diffusivity function depends on the gradient value of its magnitude. Discretizing Eq. [6] with an explicit discretization scheme ${ }^{17}$, and taking into account the image complexity and the diffusivity function modification, we come to the following numerical scheme for diffusive smoothing of complex image. Starting with an initial guess $I^{(0)}$, we proceed in a fixed number of iterations as follows:

$$
\begin{aligned}
& \operatorname{Re}\left(I_{m}^{(t+1)}\right)=\operatorname{Re}\left(I_{m}^{(t)}\right)+\Delta t \cdot \sum_{n \in \eta(m)} \nabla \operatorname{Re}\left(I_{m n}^{(t)}\right) \cdot g\left(\nabla\left|I_{m n}^{(t)}\right|, k\right) \\
& \operatorname{Im}\left(I_{m}^{(t+1)}\right)=\operatorname{Im}\left(I_{m}^{(t)}\right)+\Delta t \cdot \sum_{n \in \eta(m)} \nabla \operatorname{Im}\left(I_{m n}^{(t)}\right) \cdot g\left(\nabla\left|I_{m n}^{(t)}\right|, k\right) \\
& I_{m}^{(t+1)}=\operatorname{Re}\left(I_{m}^{(t+1)}\right)+i * \operatorname{Im}\left(I_{m}^{(t+1)}\right)
\end{aligned}
$$

Here, $\eta(m)$ is the discretization neighborhood of pixel $m$, and time step $\Delta t$ establishes the diffusion rate. The image function gradients are approximated by the nearest neighbor differences:

$$
\begin{aligned}
& \nabla\left|I_{m n}^{(t)}\right|=\left|I_{n}^{(t)}\right|-\left|I_{m}^{(t)}\right|, \\
& \nabla \operatorname{Re}\left(I_{m n}^{(t)}\right)=\operatorname{Re}\left(I_{n}^{(t)}\right)-\operatorname{Re}\left(I_{m}^{(t)}\right) \\
& \nabla \operatorname{Im}\left(I_{m n}^{(t)}\right)=\operatorname{Im}\left(I_{n}^{(t)}\right)-\operatorname{Im}\left(I_{m}^{(t)}\right)
\end{aligned}
$$

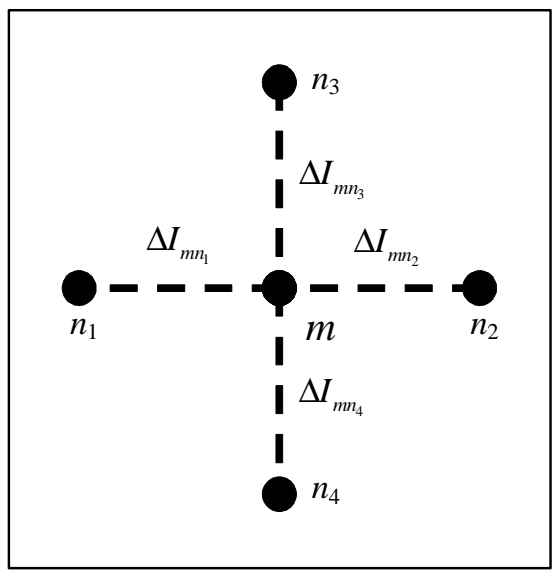

Figure 5: Four-point discretization for numerical evaluation of image gradients.

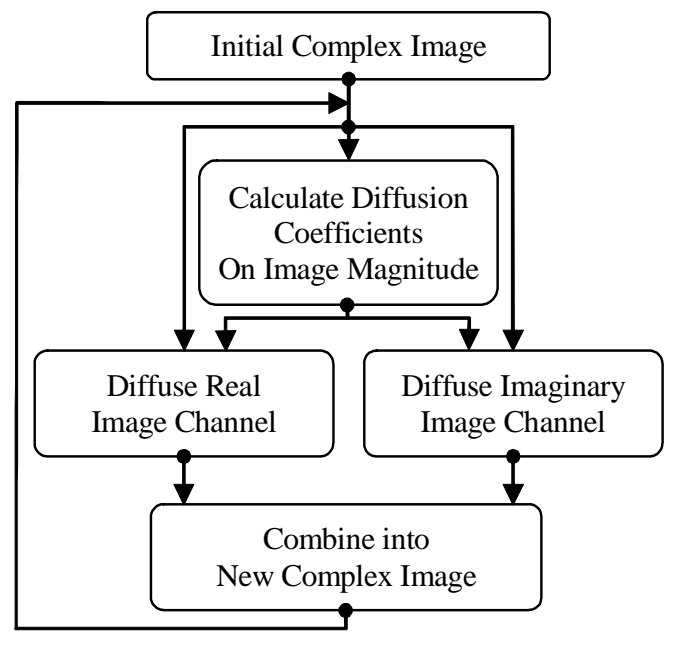

Figure 6: Flowchart of anisotropic diffusion on complex image. 
The time step $\Delta t$ is chosen using analysis of the explicit discretization scheme. We used a 4-point discretization approach (Fig. 5). In order to ensure the stability of the discretization, $\Delta t$ should be in the range of $(0,0.25]$. The diffusion scheme is illustrated in Fig. 6. In all filtering experiments, the conductance parameter was chosen based on the image noise STD. The parameter was determined using air background area samples (Eqs. $[4,5]$ ). Time step $\Delta t$ was set equal to 0.25 . The method was implemented in MATLAB 6.1 and run on a standard mid-range PC.

\section{DATA}

In order to evaluate filter performance, we created a synthetic image (256-by-256 pixels) using the well-known Shepp-Logan phantom as an image magnitude (Fig. 7a), and a linear 2D function taking values in the range $[-\pi, \pi]$ as an image phase (Fig. 7b). Then, a zero mean Gaussian noise with $\sigma=0.02$ was added to both real and imaginary image channels. The filter performance was measured by estimating the standard deviation (STD) of pixel values in the homogeneous areas of the phantom object. The root-mean-squared (RMS) error taken against the groundtruth image (Fig. 7a) was used to estimate the filtered image quality.

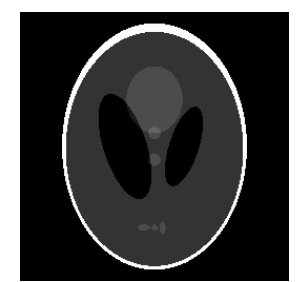

$\mathbf{a}$

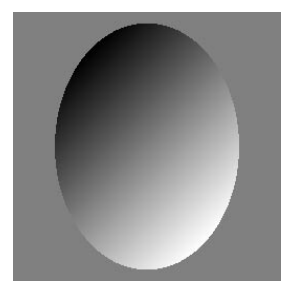

b

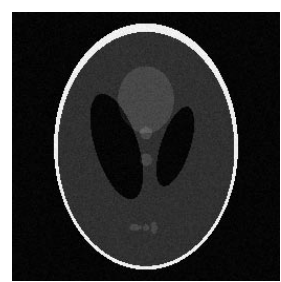

c

Figure 7: Test image. a: image magnitude, b: image phase, c: magnitude of noisy image.

The real data were obtained scanning the phantom object and patient head on a 1.5T MR scanner (GE SIGNA, GE Medical Systems, Milwaukee, WI) with a head coil using FSE acquisition protocol. Black-blood angiography data were acquired using 3D FSE protocol for black-blood imaging.

\section{RESULTS}

\subsection{Simulation studies}

Filtering experiments were performed on both image magnitude and complex images. The results are presented in Fig. 8. The newly developed technique provided significant reduction of RMS error (Fig. 8a) that corresponded to a substantial decrease in image noise (Fig. 8b). The analogous decrease in noise STD was observed for standard filtering. However, the noise elimination was not accompanied with a noticeable decrease in the RMS error. This could be explained by the fact that filtering the image magnitude left the intensity bias intact while filtering the complex image eliminated the bias (Fig. 8c). In other words, the RMS error in the image is introduced mostly by the intensity bias. Another important observation is that the significant image improvement and noise reduction could be reached using only a few iterations (10-20).

\subsection{Real data experiments}

Figure 9 shows sample profiles for the image in Fig. 4a filtered with both standard and our proposed approaches (100 iterations, $k=1.75 \sigma)$. Filtering on the complex and magnitude images produces almost identical results in high SNR areas, where bias is small. This supports the idea that filtering could be safely done separately on real and imaginary parts of the spin-echo image. However, the results differ drastically for low SNR areas, where the bias is high. The bias for our proposed method is significantly reduced. 


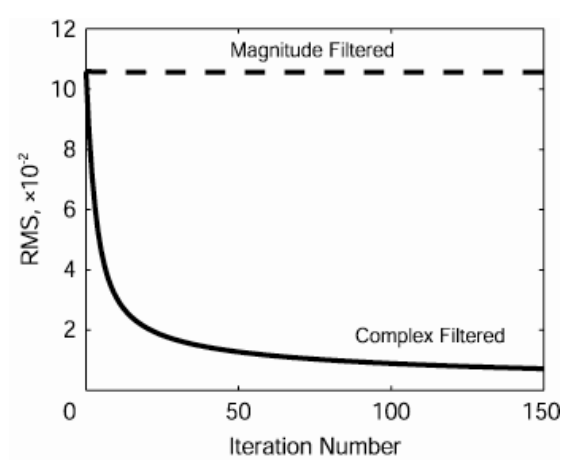

$\mathbf{a}$

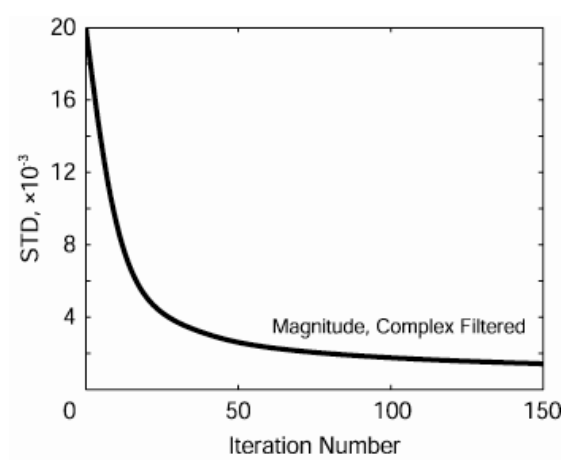

b

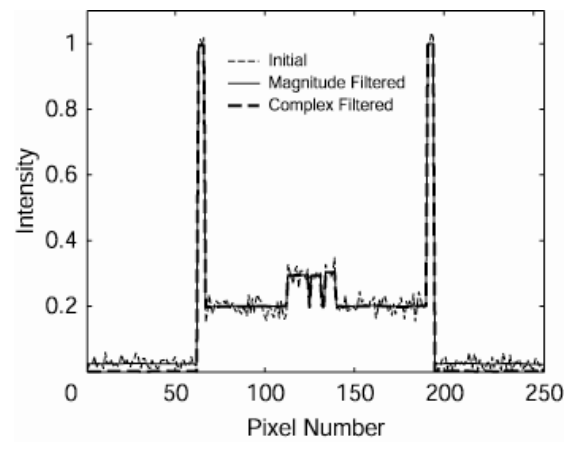

c

Figure 8: Performances of anisotropic diffusion filters on synthetic image. a: plots of RMS vs. iteration number; the change in RMS for complex filtered image is by factor 14, while that of magnitude filtered is by factor 1.003. b: plots of image noise STD vs. iteration number The plots for both filtering types are approximately the same. c: sample profiles illustrating the filter performances. Note the bias in low SNR areas for both initial and magnitude filtered images.

Figure 10 presents an example of filtering T2-weighted brain images. The filters were applied in 15 iterations with $k=1.75 \sigma$. The absolute values of differences among initial (Fig. 10a), magnitude filtered (Fig. 10b) and complex filtered (Fig. 10c) images were used to visualize the filtering results. While both filters efficiently eliminated the noise component (Fig. 10d,e), the newly developed filter produced the image with a reduced bias (Fig. 10f).

Figure 11 demonstrates results of the application of the anisotropic diffusion filters to a stack of forty 2D blackblood angiography images ( 15 iterations, $k=\sigma$ ). The brain tissues were segmented and masked before creating a Minimum Intensity Projection (MIP) image, commonly used to visualize black-blood angiography data. While both magnitude filtering and complex filtering reduce image noise, the MIP image for complex filtering has better image contrast. A prior application of our filtering method to the image stack resulted in improved black-blood data visualization.

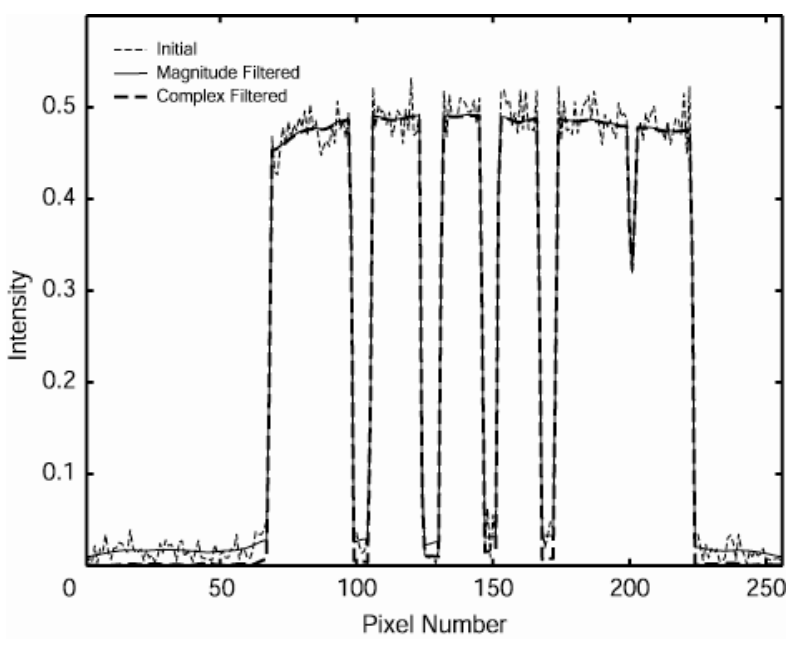

Figure 9: Filtering the phantom data. Plots of initial, magnitude filtered and complex filtered images corresponding to the profile shown in Fig. 4a are given. 


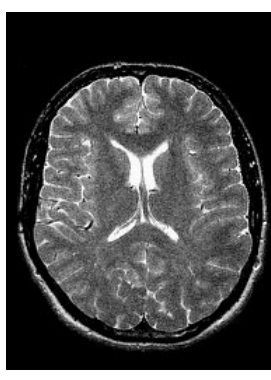

$\mathbf{a}$

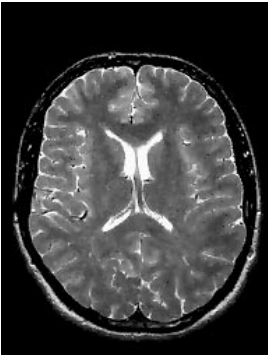

b

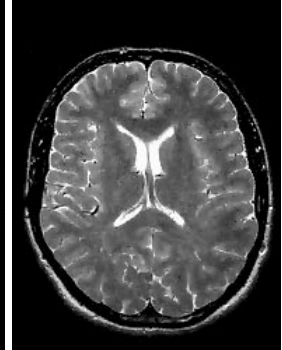

c

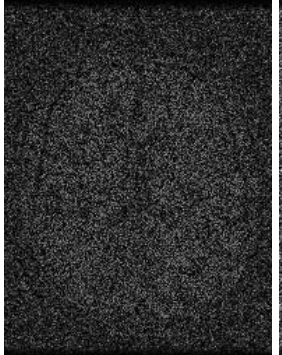

d

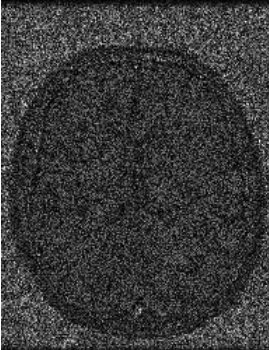

e

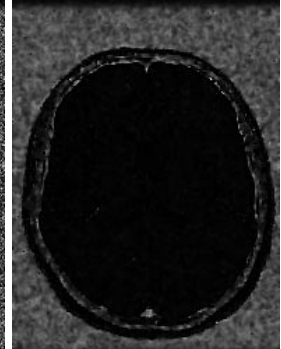

f

Figure 10: Brain image filtering. a: initial noisy image magnitude; b: filtered image magnitude; c: magnitude image after application of the proposed filter; d, e: absolute difference of $(a)$ against $(b)$ and $(c)$ correspondingly; f: absolute value of difference of $(b)$ against $(c)$. Note that the difference $(f)$ illustrates the bias eliminated using the proposed method.
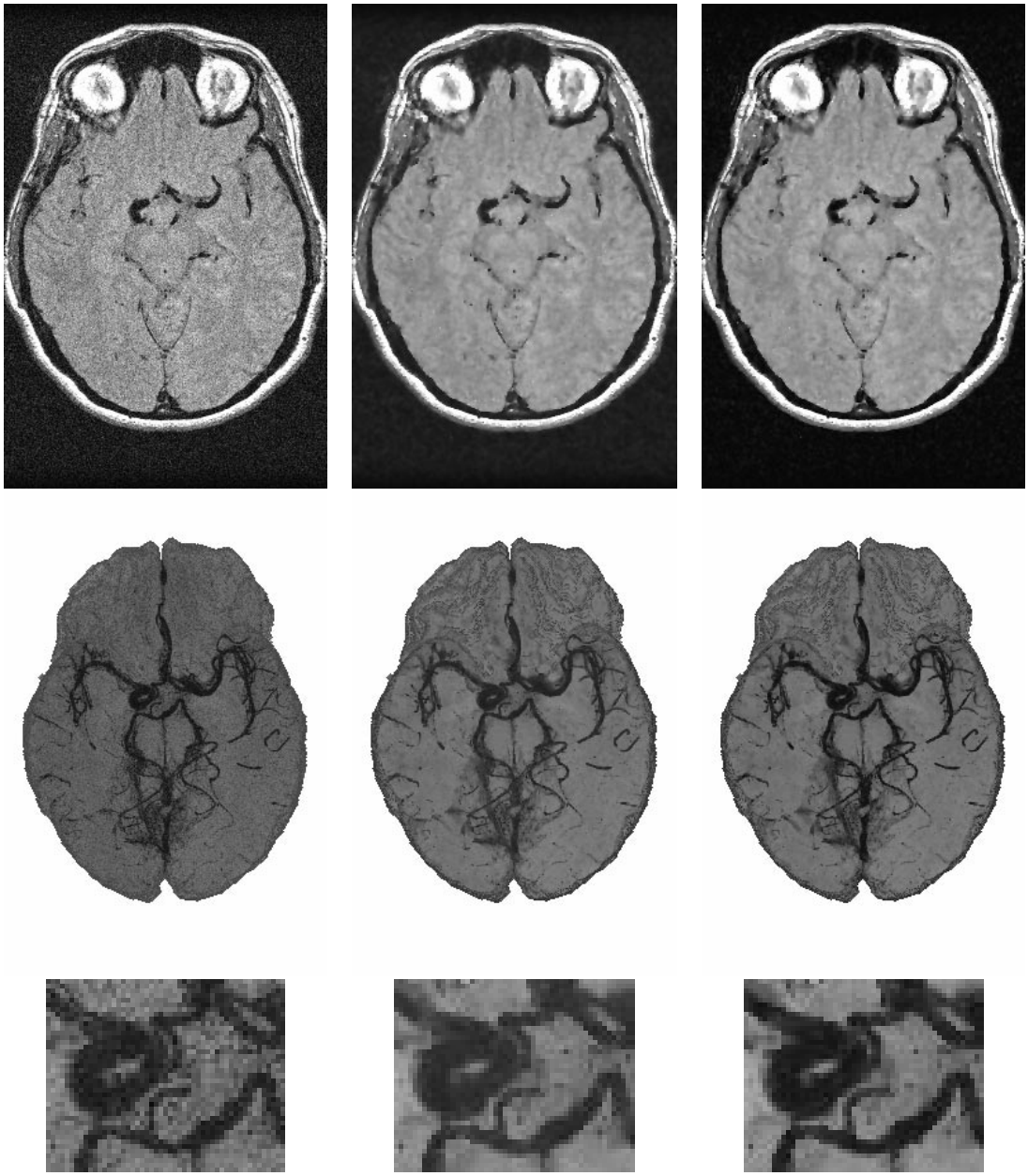

Figure 11: Black blood angiography image filtering. From left to right: initial noisy, magnitude filtered and complex filtered images. From top to bottom: single slice, MIP on the stack of segmented images, ROI of the MIP image. 


\section{DISCUSSION}

Anisotropic diffusion filtering assumes that the underlying image is piecewise constant. If the filter is applied to regions of constant slopes with a large number of iterations, it may split the regions into piecewise constant plateaus. This effect is called a staircasing artifact ${ }^{14}$. Though both real and imaginary parts of the image deviate from this model, we did not observe the described artifacts in our experiments. We attribute the behavior of the filter to the fact that the phase is slowly varying enough not to make the image channels deviate significantly from this model.

The standard time for filtering a 256-by-256 image on mid-range PC was about $0.5 \mathrm{sec}$ per iteration. We did not observe any significant filtering artifacts due to breaking the phase smoothness assumption of spin-echo MRI data. The possible explanation is that the phase variations due to blood flow and susceptibility changes usually correlate with image structures such as vessels and tissue-air boundaries. Hence, the artifacts could exhibit themselves as local edge degradation.

The application of the method to other kinds of MRI data such as data acquired with gradient echo protocols may be problematic, as the phase smoothness property is not retained in such data. Hence, the application of our proposed method is limited to spin-echo data. Further developments may include extending the scheme to filtering the image derivatives that would allow application of the filter to images that strongly deviate from a piecewise constant model. This might be particularly useful for images acquired with surface coils ${ }^{18}$ that are modulated by rapidly varying sensitivity function.

\section{CONCLUSIONS}

Intensity bias in the MRI image magnitude could present a serious problem in MRI data analysis. The removal of the bias by using filtering approaches that take into account the Rician PDF of the data is typically a numerically and algorithmically complicated procedure. Instead, we suggested using standard spatial averaging techniques that are efficient for the elimination of Gaussian noise for filtering spin-echo MRI data. The phase smoothness property allows separate filtering of both real and imaginary image parts using short scale spatial averaging.

We proposed a new method for unbiased nonlinear diffusion filtering of spin-echo MRI data. The new method was tested on a wide range of both phantom and patient data obtained using FSE acquisition protocols. Our method provided efficient image denoising and simultaneously removed an intensity bias in the image magnitude. At the same time, the new method did a good job at spatial resolution preservation enhancing image edges. The method is computationally efficient and algorithmically simple.

\section{AKNOLEDGEMENTS}

This work was supported by NIH BISTI grant 1P20HL68566-01. The authors thank Eugene Kholmovski for his assistance with imaging experiments and Ross Whitaker for his valuable comments.

\section{REFERENCES}

1. E.M. Haacke, R.W. Brown, M.R. Thompson, R. Venkatesan, Magnetic Resonance Imaging: Physical Principles and Sequence Design, 914 p., John Wiley \& Sons, New York, 1999.

2. H. Gudbjartsson, S. Patz, "The Rician Distribution of Noisy MRI Data," Magn Reson Med, 34, pp. 910-914, 1995.

3. J. Sijbers, A.J. denDekker, A. VanderLinden, M. Verhoye, D. VanDyck, "Adaptive Anisotropic Noise Filtering of Magnitude MRI Data," Magn Reson Imaging, 17, pp. 1533-1539, 1999.

4. R.R. Edelman, D. Chien, D. Kim, "Fast Selective Black-Blood MR Imaging," Radiology, 181, pp. 655-660, 1991.

5. J. Liu, A. Nieminen, J.L. Koenig, "Calculation of T1, T2 and Proton Spin Density in Nuclear Magnetic Resonance Imaging," J Magn Reson, 85, pp. 95-110, 1989.

6. Y. Wang, T. Lei, "Statistical Analysis of MR Imaging and its Applications in Image Modeling," Proceedings of the IEEE International Conference on Image Processing and Neural Networks, 1, pp. 866-870, 1994. 
7. Z.P. Liang, F.E. Boada, R.T. Constable, E.M. Haacke, P.C. Lauterbur, M.R. Smith, "Constrained Reconstruction Methods in MR Imaging," Rev Magn Reson Med, 4, pp. 67-185, 1992.

8. G.Z. Yang, P. Burger, D.N. Firmin, S.R. Underwood, "Structure Adaptive Anisotropic Filtering for Magnetic Resonance Images," Lecture Notes in Computer Science, 970, pp. 384-391, 1995.

9. P. Mrázek, "Nonlinear Diffusion for Image Filtering and Monotonicity Enhancement," PhD Thesis, Czech Technical University, Prague, June 2001.

10. P. Perona, J. Malik, "Scale-space and Edge Detection Using Anisotropic Diffusion," IEEE Trans Pattern Anal Machine Intell, 12, pp. 629-639, 1990.

11. G. Gerig, O. Kubler, R. Kikinis, F.A. Jolesz, "Nonlinear Anisotropic Filtering of MRI Data." IEEE Trans Med Imag, 11, pp. 221-232, 1992.

12. J. Sijbers, "Signal and Noise Estimation from Magnetic Resonance Images," PhD Thesis, University of Antwerpen, Antwerpen, 1998.

13. W.A. Edelstein, P.A. Bottomley, L.M. Pfeifer, "A Signal-to-Noise Calibration Procedure for NMR Imaging Systems," Med Phys, 11, pp. 180-185, 1984.

14. J. Weickert, "A Review of Nonlinear Diffusion Filtering”, Scale-Space Theory in Computer Vision, 1252 , by B. Romeny et. al., pp. 3-28, Springer, Berlin, 1997.

15. R. Whitaker, G. Gerig, "Vector-Valued Diffusion," Geometry-Driven Diffusion, by B. Romeny et al., pp. 93-134, Kluwer, Boston, 1994.

16. E.M. Haacke, E. Lindskog, “A Fast, Iterative, Partial-Fourier Technique Capable of Local Phase Recovery,” J Magn Reson, 92, pp. 126-145, March 1991.

17. G.H. Golub, Ortega J.M, Scientific Computing: An Introduction with Parallel Computing, 442 p., Boston, Academic Press, 1993.

18. P.B. Romer, W.A. Edelstein, C.E. Hayes, S.P. Souza, O.M. Mueller, "The NMR Phased Array,” Magn Reson Med, 16, pp. 192-225, 1990. 